\title{
Incidência das ametropias no Hospital Universitário em Campo Grande (MS) entre 1996 e 1998
}

\author{
Incidence of ametropias in the University Hospital of Campo Grande (MS) \\ between 1996 and 1998
}

Eduardo Velasco de Barros ${ }^{(1)}$ Vanderson Glerian Dias ${ }^{(2)}$

\section{RESUMO}

Objetivo: Avaliar o perfil epidemiológico dos casos de ametropias atendidos no Núcleo do Hospital Universitário (NHU) da UFMS, já que as pesquisas em oftalmologia nessa área são extremamente pobres em nossa região.

Métodos: Realizou-se levantamento epidemiológico retrospectivo na Seção de Arquivos Médicos (SAME), através da aplicação de protocolo de investigação de 2.361 prontuários de pacientes com algum tipo de ametropia atendidos no NHU entre 1996 e 1998, contendo sexo, idade e tipo de ametropia (miopia, hipermetropia, astigmatismo ou presbiopia). Os dados foram analisados e discutidos.

Resultados: Prevalência do sexo feminino (60\%) em todas as ametropias, o maior contingente atendido foi de pacientes com presbiopia (987 casos), seguida pela hipermetropia (701), miopia (434) e astigmatismo (239). A miopia esteve mais presente na faixa etária entre 20 e 29 anos, a hipermetropia entre 0 a 9 anos e os astigmatas entre 10 e 39 anos, enquanto a maior incidência de presbiopia foi na faixa etária acima de 40 anos.

Conclusão: O número de pacientes do sexo feminino é maior em relação ao sexo masculino, mesmo considerando cada ametropia isoladamente. Propõe-se no trabalho algumas hipóteses. A miopia concentra-se mais entre as idades de 10 a 39 anos. Já a hipermetropia ocorre mais em crianças e recém-nascidos, tendendo a diminuir com o passar da idade, devido ao aumento do globo ocular. $O$ astigmatismo foi um defeito caracterizado como sendo de adolescentes e adultos jovens. A presbiopia foi o erro de refração mais comum e sua incidência, semelhante aos dados da literatura, ocorreu na faixa etária acima de 40 anos.

Palavras-chave: Ametropia; Epidemiologia; Refração.

\section{INTRODUÇ̃̃̃O}

Emetropia é a falta de erro refrativo e as ametropias são defeitos de refração caracterizados pelos raios refratados no olho (originados de raios incidentes paralelos) não convergirem na retina (camada foto sensível) ${ }^{1-5}$. Segundo o Stedman's Medical Dictionary, ametropia vem do Grego Ametros (desproporcional) + Metron (medida) + óps (olho), ou seja, medida desproporcional do olho ${ }^{1,4}$. No caso da miopia os objetos localizados em uma distância finita do olho são focalizados na retina ${ }^{2}$. Pode ser provocada por uma ou mais das seguintes anomalias ${ }^{2}$ : 
A) AMETROPIA AXIAL: Comprimento do globo ocular, aumentado na miopia, diminuído na hipermetropia.

B) AMETROPIA DE CURVATURA: Curvatura anormal das superfícies refringentes da córnea ou cristalino, muito curva na miopia, muito plana na hipermetropia.

C) AMETROPIA DE ÍNDICE: Causada por índices anormais de refração dos meios. Na miopia de índice, o índice de refração da córnea, humor aquoso ou cristalino, é muito elevado, e do humor vítreo pode ser muito baixo. Na hipermetropia de índice ocorrem as situações contrárias, tendo o maior exemplo a afacia.

D) POSICIONAMENTO ANORMAL DO CRISTALINO: Para frente no caso de miopia, e para trás no caso de hipermetropia.

De todos esses fatores, o mais importante e o mais comum é o comprimento axial do globo ocular que, no olho emétrope, varia de 22 a $35 \mathrm{~mm}$. Os fatores genéticos têm importante papel no aparecimento das ametropias ${ }^{2,3}$.

O principal sintoma dos vícios de refração é a diminuição da acuidade visual, mas podem causar cefaléia, tontura, sonolência, hiperemia conjuntival, lacrimejamento, ardência, dor, ressecamento, etc. ${ }^{1-3,6-8}$. A diminuição do rendimento escolar, devido a problemas de refração, é um aspecto social e educacional importante e deve ser levado em consideração 9 .

As principais ametropias e as mais freqüentes são: miopia, hipermetropia, astigmatismo e presbiopia ${ }^{1-3,5}$. Entretanto, não se considera presbiopia como um erro refrativo atualmente ${ }^{6}$.

É importante que se saiba que a palavra longe em relação a acuidade visual significa que a distância de medida corresponde ao infinito óptico (6 metros). Já a acuidade de perto é obtida em geral a 30 centímetros ${ }^{10}$.

A miopia, do Grego myo (fechar) + óps (olho), ou seja, olho fechado, é o estado dióptrico do olho em que, com acomodação em repouso, os raios paralelos incidentes chegam a um foco adiante da região fotossensível da retina ${ }^{1-5,10}$.

Em casos simples de miopia geralmente não ocorrem alterações no fundo de olho, porém em casos graves de alta miopia, alterações no fundo de olho quase sempre estão presentes, inclusive o descolamento de retina ${ }^{1-3}$. A alta miopia está relacionada com maculopatia e catarata e segundo o estudo de Crucciani, metade dos pacientes com alto grau de miopia apresentam também maculopatia ${ }^{11}$.

A correção da miopia ("vista corta", em espanhol), que é mais comum nas mulheres do que nos homens, é feito pela prescrição de lentes divergentes ${ }^{1-3,5}$.

A hipermetropia ou hiperopia, do Grego hiper (aumentada) + metron (medida) + óps (olho), ou seja, medida aumentada do olho é, na verdade, o estado no qual o olho não acomodado foca a imagem atrás da retina ${ }^{4,10}$. Pode ser por redução do comprimento do eixo (olho curto) ou um problema refrativo. O hipermétrope poderá ter acuidade visual deficiente para perto, recebendo o nome em espanhol de "vista de lejos". Pequeno poder ou valor de hipermetropia está presente na maioria das crianças quando nascem. À medida que a criança cresce, esta hipermetropia, na maioria das vezes, diminui até o seu total desaparecimento ${ }^{1-5}$.

Nas primeiras décadas de vida diminui rapidamente a freqüência de hipermetropia, que persiste em mais ou menos $50 \%$ das pessoas depois dos 20 anos. Não há predomínio de sexo segundo Duke-Elder ${ }^{2}$. A correção é feita pela prescrição de lente convergente ${ }^{1-3,5}$.

O Astigmatismo é definido, segundo Vaughan ${ }^{5}$ como anormalidade no formato da córnea originando linhas ou pontos focais múltiplos. É classificado em:

A) HIPERMETRÓPICO COMPOSTO: quando nos dois meridianos oculares principais (o de maior e o de menor refração) for demonstrada hipermetropia ${ }^{1}$.

B) HIPERMETRÓPICO SIMPLES: quando num dos meridianos houver hipermetropia, sendo o outro emétrope ${ }^{1}$.

C) MISTO: quando num meridiano for achado hipermetropia e no outro, miopia ${ }^{1}$.

D) MIÓPICO SIMPLES: quando em um meridiano há emetropia e no outro, miopia ${ }^{1}$.

E) MIÓPICO COMPOSTO: quando em ambos os meridianos ocorre miopia ${ }^{1}$.

A correção é feita com o uso de lentes cilíndricas.

A Presbiopia (Presbyopia, em inglês) é a perda da acomodação visual (capacidade de mudança do poder focal do olho ${ }^{6}$ ) que vem com a idade para todas as pessoas, surgindo em torno dos 40 anos. Ocorre por perda da elasticidade da cápsula do cristalino (teoria da perda da elasticidade do cristalino de Helmholtz), o qual não mais consegue alterar sua curvatura, levando à perda da visão de perto. É corrigida pelo uso de lente positiva ${ }^{1-5}$. Recentemente tem-se outra teoria para tentar explicar a acomodação, proposta por Schachar, porém há controvérsias entre os adeptos das duas teorias. O presbíope não tem boa acuidade visual para perto ${ }^{1}$.

\section{OBJETIVO}

Descrever os casos de ametropias atendidos no Núcleo do Hospital Universitário (NHU) da Universidade Federal de Mato Grosso do Sul no período entre 1996 e 1998, relacionando a distribuição desses defeitos com sexo e idade dos pacientes, para que se tenha dados epidemiológicos de fundamental importância para a análise do volume de atendimento no ambulatório de oftalmologia, já que existem poucos trabalhos de oftalmologia com esse intuito em Mato Grosso do Sul.

\section{PACIENTES E MÉTODOS}

Foi elaborado protocolo de investigação de prontuários contendo sexo, idade e tipo de ametropia. Executou-se levantamento retrospectivo de 2361 prontuários contidos na Seção 
de Arquivos Médicos (SAME), procurando-se a notação dos dados anteriores. O tipo de ametropia foi estabelecido pelos próprios autores, por exames de acuidade visual, pela tabela de Snellen, através de refrator estático e retinoscópio no ambulatório de oftalmologia do NHU/UFMS, com cicloplegia. Não foi quantificado o erro de refração. A partir disso, os dados foram cruzados e seus resultados analisados.

\section{RESULTADOS}

Na tabela 1, observamos que do total de pacientes atendidos no NHU/UFMS (2361 pacientes), mais de 60\% (1465 pacientes) correspondem ao sexo feminino e quase $40 \%$ (896 pacientes ), ao sexo masculino. Em todos os erros refrativos pesquisados, as mulheres lideravam sempre com mais de $59 \%$ dos casos em relação a cada defeito isolado. O maior contingente atendido é, sem dúvida, os pacientes que possuíam presbiopia com 987 casos (foram selecionados os pacientes que não apresentavam erro de refração anterior ou que já haviam resolvido o defeito de refração e a partir da idade relatada começaram a apresentar dificuldade visual, além de exames oftalmológicos compatíveis com o defeito). Em segundo lugar temos a hipermetropia com 701 pacientes, em terceiro, miopia com 434 e o menor número de pacientes foi o de astigmatas com 239 casos.

Observa-se que a maior percentagem dos pacientes com miopia atendidos no NHU/UFMS (tabela 2) estão na faixa etária de 20 a 29 anos, correspondendo a $34,78 \%$ desses atendimentos, seguida pela faixa etária de 10-19, representando $20,05 \%$. Nas pessoas com 40 anos ou mais ocorre uma sensível diminuição da percentagem. Deve-se observar que a faixa etária entre 00-09 e a de 30-39 estão em nível intermediário quando relacionadas com as outras faixas.

$\begin{aligned} & \text { Tabela 1. Distribuição segundo o sexo dos casos de ametropias } \\
& \text { atendidos no NHU/UFMS entre }\end{aligned}$
\begin{tabular}{cccc} 
1996 e 1998. \\
Defeito de Refração & Homens & Mulheres & Total \\
Miopia & 174 & 260 & $\mathbf{4 3 4}$ \\
Hipermetropia & 282 & 419 & $\mathbf{7 0 1}$ \\
Astigmatismo & 94 & 145 & $\mathbf{2 3 9}$ \\
Presbiopia & 346 & 641 & $\mathbf{9 8 7}$ \\
Total & 896 & $\mathbf{1 4 6 5}$ & $\mathbf{2 3 6 1}$ \\
SAME-NHU/UFMS, 1998 & & & \\
\hline
\end{tabular}

\begin{tabular}{|c|c|c|c|c|c|c|c|}
\hline $\begin{array}{l}\text { Faixa etária } \\
(\%)\end{array}$ & $00-09$ & $10-19$ & $20-29$ & $30-39$ & $40-49$ & 50 ou + & - Total \\
\hline Homens & 5,30 & 9,68 & 12,66 & 5,99 & 2,76 & 3,69 & 40,08 \\
\hline Mulheres & 7,60 & 10,37 & 22,12 & 9,68 & 4,61 & 5,54 & 59,92 \\
\hline Total & 12,90 & 20,05 & 34,78 & 15,67 & 7,37 & 9,23 & 100,00 \\
\hline
\end{tabular}

Na hipermetropia (tabela 3 ) a faixa etária mais acometida está entre as crianças de 00 a 09 anos, com índice alto de $33,31 \%$, sendo imediatamente seguido pela faixa etária de 10 19 anos, com 18,97\%. As percentagens são quase as mesmas nas faixas etárias subseqüentes, denunciando uma uniformidade de valores.

Observando a tabela 4, nota-se que as maiores percentagens de pessoas astigmatas encontram-se entre 10 e 39 anos, com concentração maior entre as idade de 20-29 anos $(32,64 \%)$. Nas outras faixas observa-se pequenas diferenças de percentagem entre elas.

Sem dúvida a grande maioria dos presbíopes estão nas faixas etárias das pessoas com 40 ou mais anos. Deve-se, entretanto, verificar a ausência de casos entre a faixa etária de 00-19 anos e atentar-se para a percentagem de casos de presbiopia (quase 7\%) entre as idades de 20 e 39 anos (Tabela 5).

\section{CONCLUSÃO}

A amostra observada neste trabalho, 2361 pacientes, delimitou os nossos propósitos. Ao observar, porém, que o número de mulheres acometidas pelas ametropias pesquisadas era superior aos homens ${ }^{12-14}$, faz-se algumas justificativas para tentar solucionar a questão. Devido à maioria dos costumeiros e culturais trabalhos femininos (costura, tricô, crochê, etc), as mulheres se esforçam mais visualmente que os homens; fatores hormonais diferentes; o não uso de óculos pelas mulheres; fatores genéticos e outros, poder-se-ia supor a justificativa adequada aos dados encontrados. Não se encontra, entretanto, embasamento científico na literatura para esclarecer melhor essas teorias. Dos pacientes estudados, mais de 60\% (1472) correspondem ao sexo feminino e quase $40 \%$ (904), ao sexo masculino. Isso mostra o maior acometimento das mulheres quando se trata de ametropias ${ }^{12-14}$. Esta predominância tam-

\begin{tabular}{|c|c|c|c|c|c|c|c|}
\hline $\begin{array}{l}\text { Faixa etária } \\
(\%)\end{array}$ & $00-09$ & $10-19$ & $20-29$ & $30-39$ & $40-49$ & 50 ou + & - Total \\
\hline Homens & 17,40 & 6,85 & 5,14 & 3,71 & 3,14 & 3,99 & 40,23 \\
\hline Mulheres & 16,41 & 12,12 & 8,27 & 8,42 & 6,70 & 7,85 & 59,77 \\
\hline Total & 33,81 & 18,97 & 13,41 & 12,13 & 9,84 & 11,84 & 100,00 \\
\hline \multicolumn{8}{|c|}{ SAME-NHU/UFMS, 1998} \\
\hline
\end{tabular}

\begin{tabular}{|c|c|c|c|c|c|c|c|}
\hline $\begin{array}{l}\text { Faixa etária } \\
(\%)\end{array}$ & 00-09 & $10-19$ & $20-29$ & $30-39$ & $40-49$ & 50 ou + & Total \\
\hline Homens & 7,11 & 8,37 & 10,46 & 5,86 & 3,77 & 3,76 & 39,33 \\
\hline Mulheres & 3,35 & 14,22 & 22,18 & 13,81 & 2,51 & 4,60 & 60,67 \\
\hline Total & 10,46 & 22,59 & 32,64 & 19,67 & 6,28 & 8,36 & 100,0 \\
\hline
\end{tabular}




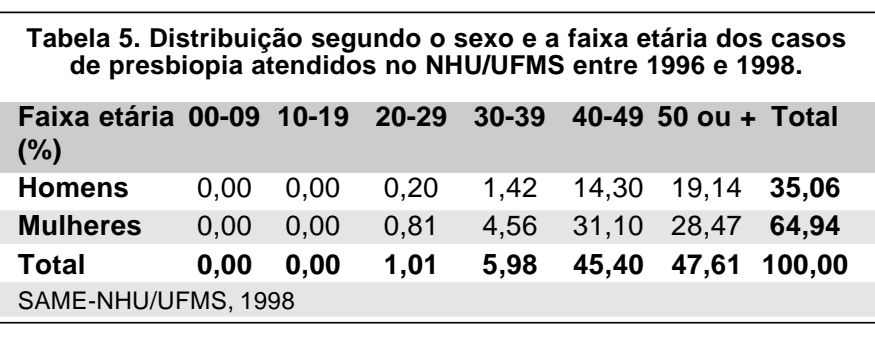

bém é verificada quando se analisa cada ametropia isoladamente, mesmos dados verificados por Kwitko ${ }^{15}$. O porquê disso pode estar mais voltado ao fato das mulheres serem mais vaidosas e não gostarem de usar óculos, já que mais de $90 \%$ delas chegam ao ambulatório do NHU/UFMS sem usá-los e por fim afirmam não se sentirem bem com o mesmo. Como, infelizmente, a lei do "uso e desuso" de "Lamark" é implacável, o contingente de mulheres com certeza será o mais atingido pois a não correção refrativa implica em piora progressiva do defeito ocular. O uso de lentes de contato corretivas e as cirurgias a "Laser" (Excimer laser, por exemplo) para correção de hipermetropia, miopia e astigmatismo, vieram substituir o uso dos óculos. Porém, não se pode contar com essa evolução toda quando se trata de Sistema Único de Saúde (SUS), já que a grande maioria dos pacientes não possui renda suficiente ${ }^{12}$ para comprar as lentes ou pagar as cirurgias refrativas, pois esses tratamentos não estão disponíveis nos hospitais credenciados pelo SUS, além da maioria desses pacientes não conhecerem métodos cirúrgicos para correção das ametroprias ${ }^{12}$.

Os resultados obtidos com o uso de PRK em pacientes com miopia leve e moderada são satisfatórios ${ }^{16,17}$, porém em pacientes com ametropias elevadas e a eficácia é menor, devido principalmente à regressão e cicatrização corneana exacerbada ${ }^{18,19}$. Tais efeitos parecem também ter relação direta com a exposição pós-operatória à radiação ultravioleta e uso de contraceptivos orais ${ }^{20}$. A PRK apresenta como alternativa para os maus resultados o retratamento; e a taxa de retratamento aumenta com a intensidade da ametropia ${ }^{21}$.

Estabeleceu-se que a miopia ocorre, em sua maior parte, entre pessoas jovens e tende a diminuir sua incidência com o passar da idade. No tocante à idade, a miopia e a hipermetropia predominam em pessoas abaixo dos trinta anos, sendo compatível com a pesquisa de Midelfart na qual a média de idade em que aparece miopia é em torno de 20 anos ${ }^{22}$. Neste trabalho, porém, diferentemente da pesquisa de Midelfart na qual não há diferença importante entre homens e mulheres, ressalta uma diferença de quase $20 \%$ a mais de mulheres comprometidas $(59,92 \%)$ em relação aos homens $(40,08)$. Do mesmo modo, a hipermetropia afeta pessoas mais jovens ${ }^{13}$, levando-se em consideração que a maior parte das crianças já nascem com hipermetropia ${ }^{1,2,5,15}$. Isso é demonstrado na faixa etária de 00-09 anos, onde foi encontrada a maior percentagem dos casos de hipermetropia $(33,81 \%)$ e pode ser explica- do já que a maioria dos bebês nascem levemente hiperópicos, e como a tendência natural é o crescimento do globo ocular, isso promove melhora do quadro chegando até a emetropia com o passar da idade, dependendo do tipo de erro refrativo hipermetrópico, por exemplo, o defeito axial ${ }^{2,5}$. Como foi observado por Dib, o número de pessoas idosas hipermétropes era maior que os de míopes ${ }^{23}$. Entretanto, os pacientes mais jovens (em torno de 20-29 anos) eram mais freqüentemente míopes ${ }^{23}$. Fantl ${ }^{24}$ realizou um extenso estudo sobre as características refracionais das crianças portadoras de paralisia cerebral e concluiu que elas apresentavam mais hipermetropia do que as crianças normais, e que a hipóxia poderia ser um fator patogênico para a hipermetropia dessas crianças ${ }^{25}$.

No caso da miopia os pacientes entre 10 e 29 anos $(54,83 \%)$ formam o maior número desse defeito. Isso se explica pelo fato que na adolescência e no adulto jovem ocorre crescimento corporal e também do globo ocular aumentando a distância do foco à retina, nas patologias do eixo. Com o passar da idade, a hipermetropia tende a diminuir sua incidência de acordo com os dados analisados e a miopia tende a aumentar, na adolescência e no adulto jovem. Nota-se um aumento significativo no número de pacientes com astigmatismo a partir dos 10 anos de idade, acompanhado por uma maior incidência na faixa etária dos 20 aos 29 anos $(32,64 \%)$. Entretanto, normalmente a criança nasce com astigmatismo considerável, e por tender a diminuir até a idade pré-escolar, na maioria das vezes não é diagnosticada promovendo uma "diminuição" no número de casos de astigmatismo em crianças, isso deve ser levado em conta. Nota-se que nesta pesquisa, o astigmatismo é um defeito essencialmente de adolescentes e adultos jovens.

Em muitos estudos envolvendo pacientes altos míopes, tem-se observado diminuição da espessura corneana ${ }^{26-28}$, além de astigmatismo associado ${ }^{26}$.

A presbiopia, sem dúvida, afeta mais os pacientes acima dos 40 anos $(93,01 \%)$, apesar de alguns pacientes apresentarem uma variação precoce do defeito entre as idades de 20 e 39 anos $(7,00 \%)$, que não se trata de alta hipermetropia já que os pacientes não apresentavam ou haviam corrigido defeitos de refração anteriores à presbiopia. Esses casos de presbiopia precoce ocorreram em nosso trabalho, devido a problemas variados, entre eles a fadiga muscular devido ao excesso de trabalho visual e acomodativo ${ }^{1-2,}$ 5,6. Deve-se observar a ausência de casos de presbiopia precoce abaixo de 20 anos, dado observado tanto na literatura quanto neste trabalho ${ }^{29}$. É importante salientar-se que na hipermetropia não corrigida, a presbiopia aparece mais precocemente do que no emétrope, o que reforça os casos de presbiopia precoce, excluídos neste trabalho. Em termos de diagnóstico de presbíopes, levou-se em consideração o grande número de pessoas com idade acima de 40 anos no consultório de oftalmologia do NHU/ UFMS.

A presbiopia foi a ametropia mais incidente entre os pacientes estudados (987), seguida pela hipermetropia (701), 
mio-pia (434) e astigmatismo (239), diferentemente dos dados obtidos por Kwitko nos quais a hipermetropia aparecia em primeiro lugar ${ }^{15}$. Já na pesquisa de Chow, a miopia e o astigmatismo possuem as maiores porcentagens ${ }^{29}$.

Deve-se salientar ainda que os defeitos refrativos são herdadas através de herança complexa envolvendo muitas variáveis ${ }^{5}$ e por isso muitas vezes com incidências não tão bem conhecidas e nem tão pouco esclarecidas.

Entre as patologias oftálmicas, aquelas cujo processo de formação é o degenerativo são muito comuns em oftalmologia. Pode-se citar a catarata senil ou diabética, retinopatia diabética e a presbiopia que neste trabalho foi a que mais acometeu pacientes.

A acuidade visual varia com a idade ${ }^{30,31}$. Contudo, se em relação à queda da magnitude do poder resolutivo que ocorre na senescência existe um razoável consenso ${ }^{32-35}$, as características quantitativas de desenvolvimento da acuidade visual durante a infância são ainda objeto de controvérsia ${ }^{10}$. A literatura clássica admite níveis de resolução muito baixos após o nascimento e um desenvolvimento lento cuja maturação plena (acuidade igual a um) só ocorreria aos cinco anos ${ }^{35,36}$. Porém, modernamente, estudos com metodologia objetiva, nistagmo optocinético, olhar preferencial visual evocado ou provocado, admitem que a acuidade da criança seja bem melhor do que se supunha ${ }^{10}$. Medidas com o olhar preferencial indicam que com 1 ano já se possua um poder resolutivo igual a $20 / 40{ }^{10}$. Tais resultados não são unanimente aceitos ${ }^{37}$. $\mathrm{O}$ trabalho de Frisén \& Frisén ${ }^{38}$ mostra que há um crescimento da acuidade visual até os 25 anos e um declínio gradual após esta idade. Tal declínio é maior após 60 anos de idade.

Ocorreram alguns dados discordantes entre este trabalho e a literatura previamente publicada, os quais podem ser explicados por fatores genéticos diferentes em relação às amostras observadas nos diferentes trabalhos, número reduzido de pacientes em algumas pesquisas abordadas e nível sócio-econômico-cultural diferente quando se trata de população amostral de outras pesquisas com o mesmo intuito.

\section{SUMMARY}

Purpose: To assess the epidemiological profile of the ametropia cases attended at the University Hospital Nucleus (NHU) of the Federal University of Mato Grosso do Sul (UFMS) since ophthalmologic research in this are is extremely poor in our region.

Methods: A retrospective epidemiological surgery at the Section of Medical Files (SAME) was carried out, applying a research protocol to 2,361 charts of patients presenting some type of ametropia who were attended at the NHU between 1996 and 1998, with information on sex, age and type of ametropia (myopia, hyperopia, astigmatism or presbyopia). The data were analyzed and discussed.

Results: There was prevalence of females (60\%) in all cases of ametropia, the greatest number of attended people being patients with presbyopia (987 cases), followed by hyperopia (701), myopia (434) and astigmatism (239). Myopia was present mostly in the age range of 20 to 29 years, hyperopia between 0 and 9 years and astigmatism between 10 and 39 years, while the highest incidence of presbyopia was in the age range over 40 years.

Conclusion: The number of female patients is higher than that of males, even considering each ametropia separately. Some hypotheses are proposed. Myopia is more frequent at ages between 10 and 39 years. Hyperopia occurs more frequently in children and newborns, tending to decrease with age because of eyeball increase. Astigmatism was characterized as a defect occurring in adolescents and young adults. Presbyopia was the commonest refraction error and its incidence occurred in the age range over 40 years, similarly to data found in the literature.

Keywords: Ametropia; Epidemiology; Refraction.

\section{REFERÊNCIAS BIBLIOGRÁFICAS}

1. Bicas HEA. Oftalmologia: Fundamentos. $1^{\mathrm{a}}$ ed. São Paulo. Editora Contexto 1991;2 e 3:89-92 e 141-58.

2. Duke-Elder. Enfermidades de los ojos. $15^{\mathrm{a}}$ ed. Mexico. Nueva Editorial 1971;6-9:47-81.

3. Porto CC. Semiologia Médica. $2^{\text {a }}$ ed. Rio de Janeiro. Editora Guanabara Koogan S.A. 1994;12-14:169-202.

4. Stedman'S. Medical Dictionary. 26 $6^{\mathrm{a}}$ ed. Baltimore. Williams \& Wilkins. $1995 ; 159,828,1170-422$.

5. Vaughan DG, Asbury T, Riordan-Eva P. Oftalmologia Geral. $4^{\mathrm{a}}$ ed. São Paulo. Editora Atheneu LTDA. 1998;20:375-80.

6. Bicas HEA. Ametropias e presbiopia. Medicina (Ribeirão Preto). 1997;30(1):20-6.

7. Gelvez V. La cefalea y su relacion com los defectos de refracion. Rev Oftalmol Venez 1982;40(3):247-53.

8. Lauretti AF, Ramão E. Vícios de refração e cefaléia habitual em crianças. Rev Bras Oftalmol 1987;46(2):27-31.

9. Guerrero RV, Martinez C, Carmen NE, Woolley L. Defectos de refracion y rendimiento academico en la escuela primaria. Colomb méd 1989;20(1):8-10.

10. Rodrigues MLV et al. Oftalmologia Clínica. $1^{\text {a }}$ ed. Rio de Janeiro, Editora Cultura Médica LTDA. 1992;6 e 7:72-114.

11. Crucciane F, Lombardo E, Maglione A, Senderi G. Relación entre maculopatia y catarata en pacientes miopes elevados. Rev Oftalmol Venez 1991;47(4):31-5.

12. Moreira H, Maia M, Maia EM, Godoy G, Huss HHA. Aspectos sôcio-econômicos do uso dos óculos no Hospital de Clínicas da UFPR. Arq Bras Oftal 1997:60(4):354.

13. Cavalcanti SJL, Lopes RACE, Carvalho RF. Anomalias refrativas em população escolar. Arq Bras Oftal. 1996;59(4):352.

14. Sales SG, Pessoa SAR, Carvalho RF, Trigueiro SA, Bueno LG. Afecções oculares encontradas em pacientes da periferia de Jaboatão dos Guararapes (PE). Arq Bras Oftal 1998;61(4):434.

15. Kwitko S, Kwitko Il. Casuística dos erros de refração no Hospital das Clínicas de Porto Alegre. Rev AMRIGS 1984;28(4):318-24.

16. Snibson G, Carson C, Aldred G, Taylor H. One-year evaluation of excimer laser photorefractive keratectomy for myopia myopic astigmatism. Arch Ophthalmol 1995;113:994-1000.

17. Dutt S, Steinert R, Raizman M, Puliafito C. One year result of excimer laser photorefractive keratectomy for low to moderate myopia. Arch Ophthalmol 1994; 112:1427-36.

18. Krueger R, Talamo J, McDonald M, Varnell R, Wagoner M, McDonnel P. Clinical analysis of excimer laser photorefractive keratectomy using a multiple zone technique for severe myopia. Am J Ophthalmol 1995;119:263-74.

19. Seiler T, Derse M, Pham T. Repeated excimer laser treatment after photorefractive keratectomy. Arch Ophthalmol 1992;110:1230-3.

20. Corbett M, O'brart D, Warburton F, Marshall J. Biologic and environmental 
risk factors for regression after photorefractive keratectomy. Ophthalmology 1996; 103:1381-91

21. Vázquez M, Schor P, Chamon W, Allemann N, Campos M. Retratamento em cirurgias refrativas com excimer laser. Arq Bras Oftal 1998;61(1):82-6.

22. Midelfart A, Aamo B, Siahang KA, Dysthe BE. Myopia among medial students in Norway. Acta Ophthamol (Copench) 1992;70(3):317-22.

23. Dib A. Distribution of retractive errors in patients from Dominica, West Indies. J Am Optom Assoc 1990;61(1):40-3.

24. Fantl EW, Perlstein MA. Ocular refractive characteristics in cerebral palsy. Am J Dis Child 1961;102:36.

25. Tabuse MKU, Moreira JBC. Estudo das manifestações oculares em crianças com paralisia cerebral. Arq Bras Oftal 1996;59(6):560-6.

26. Tanaka HM, Mori ES, Maia NCF, Juliano Y, Campos M. Espessura corneana na alta miopia. Arq Bras Oftal 1996;59(3):295-8.

27. Santoni A. Sullo Spessore Della Cornea in caso di miopia elevata. Rass Ital Attal 1952;21:219.

28. Tokoro T, Hayashi K, Muto M et al. Central corneal thickness in high myopia. Folia Ophthalmol Jap 1976;27:610.
29. Chow YC, Dhillon B, Chew PT, Chew SJ. Refractive errors in Singapore medical students. Singapore med J 1990; 31(5):472-3.

30. Catford GV, Oliver A. Development of visual acuity. Arch Diseas Childhood $1973 ; 48: 47-50$

31. Visual acuity. Ann Rev Psychol 1965;16:359-81.

32. Chapanis A. Relationship between age, visual acuity and color vosion. Human Biology 1950;22:1-33.

33. Milne JS, Willianson J. Visual acuity in older people. Geront Clin 1972;14:249-56.

34. Pollock FJ. Visual acuity in the aged. J Am Ger Soc 1966;14:299-300.

35. Slapater FJ. Age norms of refraction and vision. Arch Ophthalmol 1955;43:704-25.

36. Sarux H, Biais B. Physiologie de l'acuité visuelle. Physiologie Oculaire. Paris. Masson 1973;12:230-40.

37. Fledelius H. Visual acuity (Prematurity and the eye). Acta Ophthalmologica Suppl 1976;128:113-25.

38. How good is normal visual acuity? Alb V Graf Arch Klin Exp Ophthalmol 1981;215:149-57.

\section{ENCONTRO ANUAL DA ACADEMIA AMERICANA DE OFTALMOLOGIA}

\section{1 a 25 de Outubro de 2000}

\section{DALLAS - TX}

Informações: American Academy of Ophthalmology

P.0. Box 7417 - San Francisco - CA 94120-7424

Tel.: (415) 561-8500 • Fax: (415) 561-8533

A home page da Academia, com o respectivo e-mail, pode ser
acessada a partir da home page do CBO:
http://www.cbo.com.br

